Revue scientifique sur la conception et l'aménagement de l'espace

$8 \mid 2012$

Les concepteurs de jardins et de parcs japonais

\title{
Évolution des modèles d'implantation de la villégiature sur le littoral tunisois
}

Resorts's implantation on the coastline of tunis: Models and factors of evolution

\section{Sondès Zaier, Hichem Rejeb et Pierre Donadieu}

\section{(2) OpenEdition}

\section{Journals}

Édition électronique

URL : http://journals.openedition.org/paysage/15921

DOI : 10.4000/paysage. 15921

ISSN : 1969-6124

\section{Éditeur :}

École nationale supérieure du paysage de Versailles-Marseille, Institut national des sciences appliquées Centre Val de Loire - École de la nature et du paysage, École nationale supérieure d'architecture et de paysage de Bordeaux, École nationale supérieure d'architecture et de paysage de Lille, Agrocampus Angers

Référence électronique

Sondès Zaier, Hichem Rejeb et Pierre Donadieu, «Évolution des modèles d'implantation de la villégiature sur le littoral tunisois », Projets de paysage [En ligne], 8 | 2012, mis en ligne le 09 octobre 2012, consulté le 11 mars 2021. URL : http://journals.openedition.org/paysage/15921 ; DOI : https:// doi.org/10.4000/paysage. 15921

Ce document a été généré automatiquement le 11 mars 2021.

Projets de paysage 


\section{Évolution des modèles d'implantation de la villégiature sur le littoral tunisois}

Resorts's implantation on the coastline of tunis: Models and factors of evolution

Sondès Zaier, Hichem Rejeb et Pierre Donadieu

\section{Introduction}

1 Selon le dictionnaire Le Robert (Rey, 2005), le mot villégiature, apparu en 1728, est emprunté à l'italien villégiatura et signifie "séjour à la campagne ou dans un lieu de plaisance (ville d'eaux, plage...)»; Ce terme désigne à la fois la pratique liée aux vacances et en même temps le lieu de séjour. À l'instar des riches Italiens et des souverains du XvI ${ }^{\mathrm{e}}$ siècle qui se firent construire des maisons de plaisance (Boyer, 2008), les beys ${ }^{1}$ tunisiens multiplièrent les palais de plaisance autour de la ville de Tunis à la même époque. Depuis, leur nombre ne cesse d'augmenter. La villégiature littorale concerne aujourd'hui un nombre de plus en plus important de Tunisiens et contribue à des changements profonds des paysages littoraux. Les conséquences se font aujourd'hui sentir sur le plan social, économique, environnemental et paysager, car les processus d'urbanisation se mettent en place de façon largement spontanée, avec une extension très rapide et une absence de planification préalable.

2 Le dernier recensement établi par l'Institut national de statistique tunisien montre que le nombre de ces résidences est passé de 58500 en 1994 à 83515 en 2004. Il ne s'agit donc pas tant d'un phénomène nouveau que d'un processus en pleine expansion. Le manque de planification des usages des sols des territoires où se construisent les résidences estivales pose un problème au niveau de l'occupation du sol.

Cet article, qui s'appuie sur un travail de thèse de doctorat ${ }^{2}$, propose de comprendre les mécanismes de ce développement urbain spécifique, afin de mieux anticiper son évolution future. Pour cela, nous analyserons les processus de son émergence, de son 
évolution et de ses mutations, depuis ses origines jusqu'à nos jours. Nous essaierons de montrer les résultats des analyses faites à partir de cartes anciennes ou de photographies aériennes, et de documents d'archives et d'observations de terrain. La zone étudiée est celle de la région de Tunis, lieu de l'émergence de la villégiature balnéaire en Tunisie (figure 1). Nous y décrirons l'évolution, à travers l'histoire, des modes d'implantation territoriale des habitations dédiées à la villégiature. L'enjeu est d'expliquer en quoi l'urbanisation de villégiature n'est ni anarchique ni informelle, mais qu'elle résulte de mécanismes sociaux mettant en œuvre une hiérarchie complexe de l'espace balnéaire. Notre objectif principal est d'éclairer la naissance d'une pratique de loisirs qui donne une indication du changement des relations des Tunisiens avec la mer et son rivage

Figure 1. Localisation des stations et des lieux de villégiature au début du $x^{e}$ siècle dans la région de Tunis

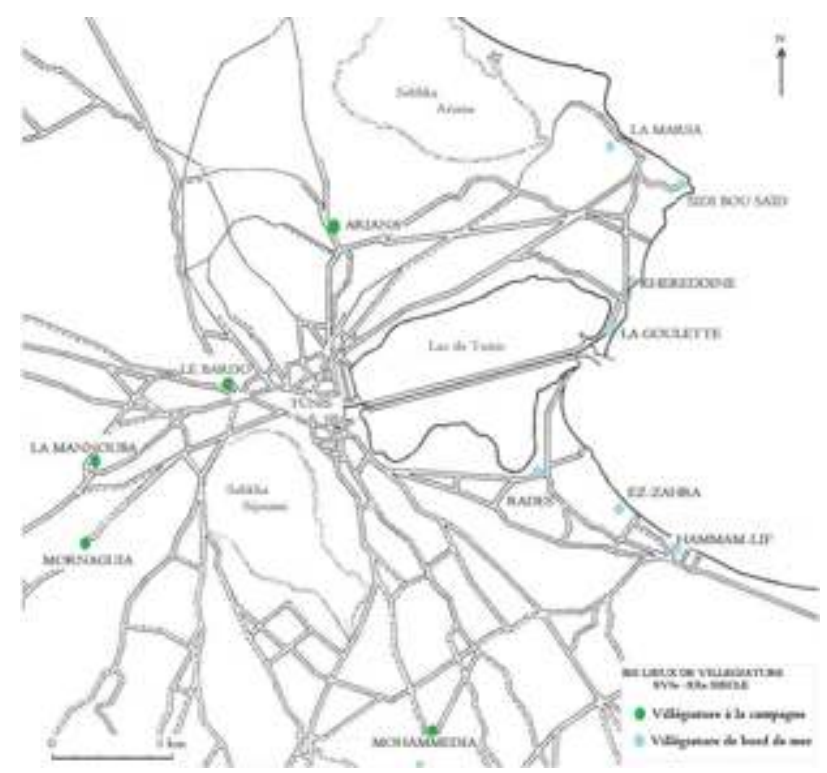

Source : Revault, J., Palais et demeures de Tunis ( $x v I^{e}$ et xvII siècles), Paris, Éditions du Centre national de recherches scientifiques, 1980. Carte actualisée par Sondès Zaïer.

\section{La construction du désir tunisien de villégiature}

La pratique de la villégiature en Tunisie trouve ses origines vers le $\mathrm{xvI}^{\mathrm{e}}$ siècle, lorsque les classes aristocratiques hafsides ${ }^{3}$ transforment les lieux de pèlerinage, les marabouts, en lieux de séjour estival. Le choix des sites n'est autre que celui opéré plus avant par les religieux ${ }^{4}$, principalement soufistes, en quête de lieux de méditation et enclins à préférer les collines de bord de mer propices à la contemplation (Ben Achour, 2004). Ces saints personnages cherchaient toujours les sommets des collines pour se livrer à la méditation et à la prière (Zbiss, 1971). C'est ainsi que plusieurs d'entre eux ont choisi le littoral de la banlieue nord de Tunis pour se retirer (figure 2). Ainsi, ils furent pendant longtemps les seuls occupants des campagnes côtières (Mahjoub, 1999). 
Figure 2. Vue de la baie de Tunis à partir de la zaouïa de Sidi Belhassen

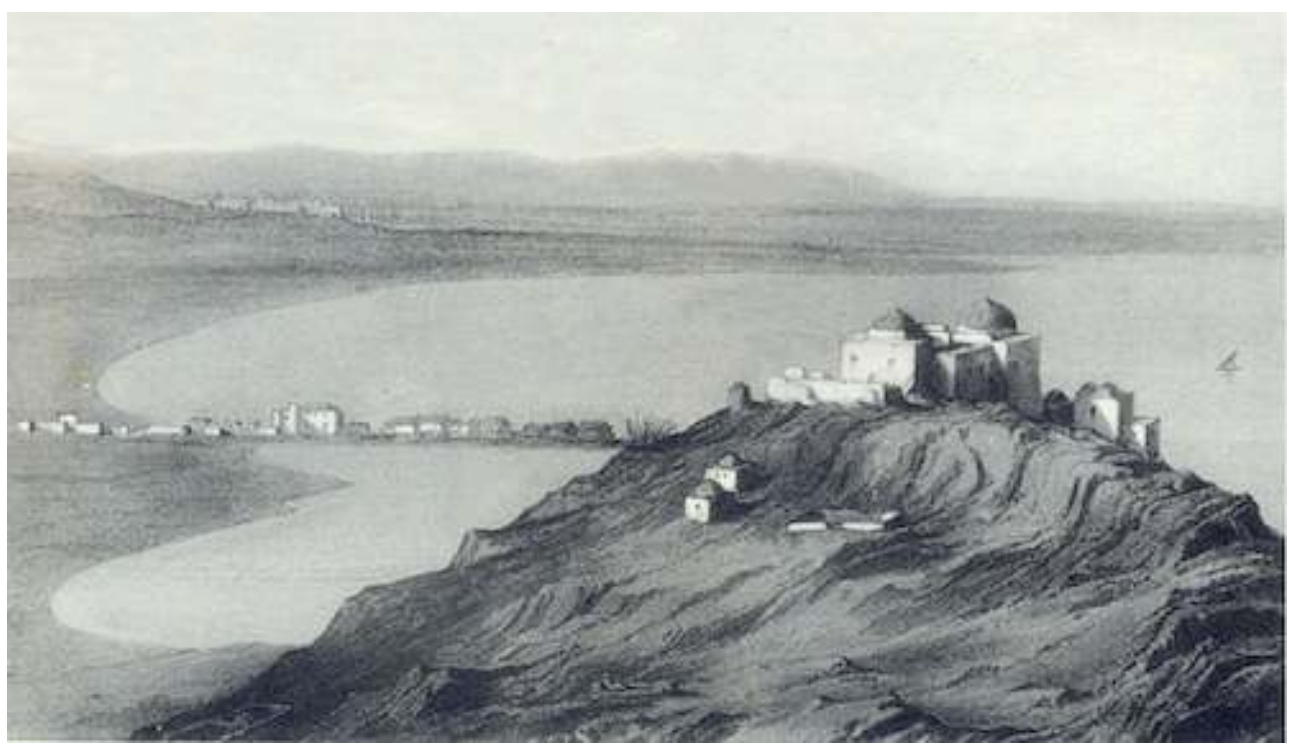

Source: Gravure de Charles de Charisson, Aperçu pittoresque de la Régence de Tunis, Paris, Imprimerie Bénard \& Cie, 1849.

$5 \quad$ Une fois installés, les princes hafsides créèrent des lieux de plaisance convoités par les dignitaires de l'État et les fonctionnaires de haut rang. La construction des palais de plaisance débuta dès le milieu du XIII ${ }^{\mathrm{e}}$ siècle avec le palais de Ras Et-Tabia qui a été décrit par plusieurs voyageurs (Brunschvig, 2001). Ce mouvement se poursuivit et les palais de plaisance se déplacèrent de la campagne vers le littoral. Le premier palais de plaisance littorale fut construit à La Marsa au début du XvI ${ }^{\mathrm{e}}$ siècle et appelé El-Abdelliya (L'Africain, 1517/1956). Depuis, ce palais devint résidence d'été de plusieurs souverains hafsides comme l'affirme Robert Brunschvig: «Avec la construction de El-Abdelliya s'institua pour le souverain l'usage de passer l'été à La Marsa. »

Les sources historiques qui donnent une idée sur l'occupation de la côte à cette époque sont rares. Nous avons trouvé un témoignage précieux datant de 1517, du voyageur Léon l'Africain, qui décrit La Marsa à cette époque comme étant déjà un lieu de villégiature :

«C'est une petite ville ancienne bâtie sur le bord de mer. C'est là que se trouvait le port de Carthage [...]. Mais elle est aujourd'hui habitée par des pêcheurs, cultivateurs et blanchisseurs de toiles. Il existe près d'elle des palais royaux et des propriétés où le roi actuel a coutume de passer tout l'été.»

Plus tard, les beys de Tunis contribuèrent à répandre cette pratique sur toutes les côtes de la région de Tunis (Dunant, 1958). Ils se sont souvent eux-mêmes inspirés, tant dans la forme de leurs résidences que dans le choix de leurs emplacements, des pratiques déjà mises en œuvre par les Hafsides.

Les princes de la famille husseinite, les consuls européens et les riches familles tunisiennes faisaient bâtir dans les environs de ce palais d'autres villas et demeures princières, selon les mêmes motivations que les beys (Revault, 1980). 


\section{Naissance des pratiques balnéaires}

9 Le XIX siècle européen fut marqué par l'émergence puis la diffusion d'une nouvelle pratique : la villégiature balnéaire. Les déplacements de la bourgeoisie sont, dans un premier temps, motivés par des raisons thérapeutiques. Peu à peu, la villégiature devint ce qu'elle est aujourd'hui, un séjour de plaisance et de repos accompagné de nouveaux loisirs comme la baignade (Corbin, 1988).

10 En Tunisie, le phénomène balnéaire apparaît à la fin du XIX $X^{e}$ siècle. Le goût du rivage est issu de la famille beylicale elle-même et de la bourgeoisie. Progressivement, il engendrera d'autres formes d'usage de la plage. La conviction, chez les classes aisées, que le changement d'air est nécessaire pour rester en bonne santé est le fondement sur lequel s'établit la motivation qui amène les premiers beys husseinite à se rendre dans des installations balnéaires appelées bit el bhar (figure 3).

11 Si « la crainte de la mer et la répugnance pour le séjour sur ses rivages » (Corbin, 1988) sont des sentiments qui, globalement, dominent toujours aux XVII et XVIII ${ }^{e}$ siècles, JeanDidier Urbain (2002) démontre que ces attitudes négatives, quoique moins explicites, sont encore fort présentes dans la sensibilité collective jusqu'à l'aube du $\mathrm{xx}^{\mathrm{e}}$ siècle. Il semble que c'est pour cette raison que les premiers palais de plaisance étaient construits en retrait par rapport à la mer. Ce qui n'empêchait pas la famille régnante d'aller se baigner. C'est ainsi qu'elle se faisait construire des cabines de mer comparables à celles qui apparaissaient au sud de l'Angleterre, notamment à Brighton. Ces constructions garantissaient une intimité familiale qui permettait aux femmes de se baigner loin des regards indiscrets.

Figure 3. Le bain des femmes du bey, à La Marsa

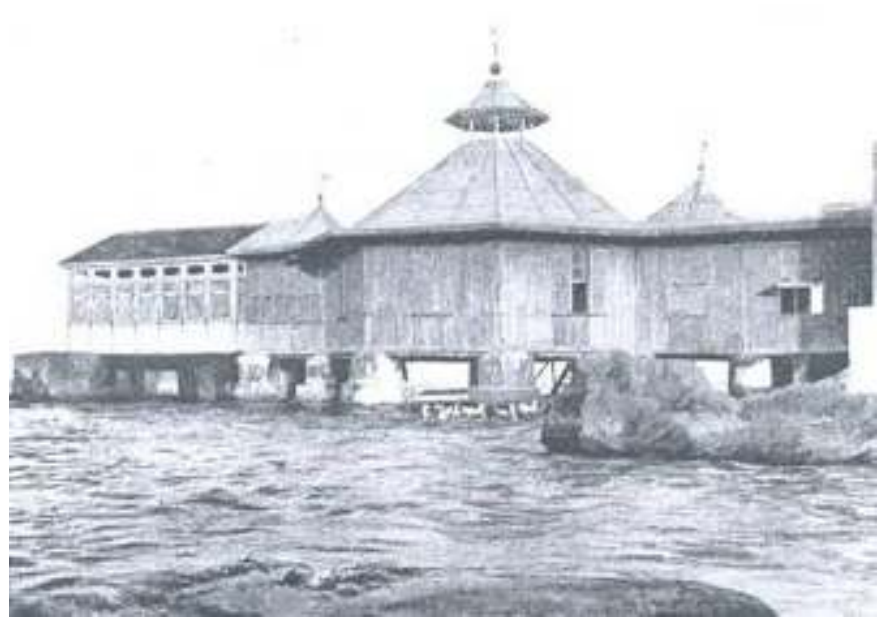

Source : Myriame Harry, «Plages tunisiennes », L'Illustration, n 3464, 17 juillet 1909, p. 42.

12 Avec la colonisation française, l'organisation du temps change fondamentalement et de nouvelles formes de loisirs apparaissent dont le premier élan reste lié aux apports sociocultuels des Ottomans et aussi aux contacts avec les pays de la Méditerranée comme l'explique Dalenda Largueche (2004):

«La colonisation va accélérer une tendance déjà amorcée où les loisirs et les

divertissements se sont réservé leurs espaces et leurs usages. » 
13 C'est ainsi que la villégiature tunisienne passe d'une pratique élitiste à celle de masse au cours de la première moitié du xx siècle (Bergaoui, 2005). Dès lors le nombre des stations balnéaires augmente, et leur nature change au fur et à mesure qu'évolue la place occupée par les loisirs. L'influence du tourisme international vient ensuite progressivement se greffer sur ce qui s'est d'abord construit comme une pratique multiculturelle mais spécifiquement tunisienne ${ }^{5}$.

\section{Les modèles d'urbanisation des lieux de villégiature littorale. Démarche d'analyse}

14 L'organisation spatiale des premières stations implantées sur le littoral de la baie de Tunis correspond à des formes de construction réfléchies. La lecture des plans géométriques qui les caractérisent offre un contraste saisissant avec les stations balnéaires actuelles. L'organisation des premiers lieux de villégiature autour de l'élément central, qui est la mosquée, cède la place à d'autres formes d'organisations urbaines. Les nouveaux tracés favorisant la vue sur l'horizon annoncent, désormais, l'avènement des fronts de mer. Le casino puis les grands hôtels y occupent dès lors la position centrale traditionnellement réservée à la mosquée. Plusieurs modèles apparaissent.

15 Selon l'architecte-urbaniste français Christian Devillers (2004), les modèles en architecture et en urbanisme permettent de rendre compte de l'attribution de valeurs aux espaces, c'est-à-dire de la façon particulière dont un groupe social se représente les propriétés de l'espace en fonction de sa propre pratique ou de ses objectifs sur l'espace. Les modèles organisent, ainsi, la pratique spatiale des usagers et celle des villégiateurs dans notre cas.

16 Ce travail de modélisation est né d'un constat: la présence, autour de Tunis, de nombreuses stations balnéaires de style différent bien qu'aménagées durant la même époque, entre la fin du $\mathrm{XIX}^{\mathrm{e}}$ et celle du xxe siècle. Chacune de ces stations sera dès lors analysée de plus près (date de création, créateur, les premiers installés, le style architectural, le tracé urbain,...)

17 Il nous a fallu pour ce travail de typologie revenir en arrière et recomposer le site initial de la future station ou du futur lieu de villégiature, depuis la date des premières installations jusqu'aux flux des villégiateurs. Le fait que les anciens lieux de villégiature sont devenus aujourd'hui des lieux de résidence permanente nous a conduit à travailler sur les anciens plans qui permettent d'analyser de plus près les premières implantations jusqu'à la formation d'un premier noyau balnéaire.

18 Cette étude des différentes stations balnéaires et des lieux de villégiature de la région de Tunis nous a permis de dégager trois modèles d'urbanisation : le modèle «familial », le modèle traditionnel, et le modèle importé.

\section{Le modèle familial}

19 Ce premier modèle est apparu au XIX ${ }^{\mathrm{e}}$ siècle à La Marsa. Les résidences secondaires sont construites autour des grands jardins qui sont aménagés en vergers. Chaque parcelle délimitée sur le plan (figure 4) représente la propriété d'une famille. Dans chaque parcelle nous trouvons plus d'une résidence secondaire. C'est le cas pour le domaine de 
la Couronne où il y avait autrefois plusieurs palais de beys et de princes de la famille husseinite.

L'identification des propriétaires de ces résidences et l'analyse du plan cadastral datant de 1978 montrent que l'agencement territorial des zones de villégiature reproduit sous forme spatiale l'organisation sociale des villégiateurs eux-mêmes: le «désir de villégiature " apparaît d'abord chez un précurseur (généralement le bey ou un bourgeois tunisois) avant d'être transmis à son environnement familial et social. Nous constatons ainsi que cette zone de villégiature n'est pas une simple juxtaposition de villas individuelles, mais qu'elle recompose dans l'espace la structure de la famille étendue ${ }^{6}$.

Figure 4. Détail du domaine de la couronne, d'après un extrait du plan de La Marsa en 1906

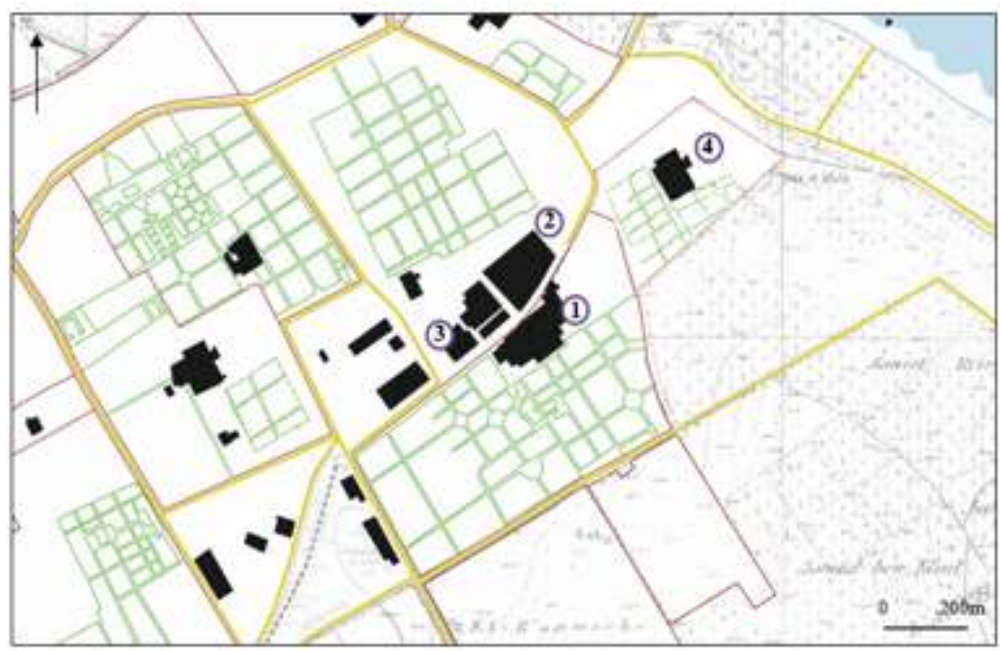

(1) Ali Bey fut le premier à s'installer dans le palais de Dar Et-Taj entre 1882 et 1892, ses trois fils avaient plus tard fait construire sur la même propriété trois autres palais de plaisance : le premier celui de Ahmed Bey (2), ensuite celui de Mustapha Bey (3) et enfin celui de Ismaill Bey (4). Plus tard, c'est tout un quartier princier qui voit le jour avec la même organisation familiale des résidences d'été.

L'analyse du tracé général montre que l'implantation initiale de toutes ces résidences secondaires avait suivi la trame agraire tracée par les Romains comme l'illustre l'extrait de la carte dessinée par Falbe en 1831 ci-dessous (figure 5). 
Figure 5. Les axes en jaune représentent le cadastre romain adopté par les beys husseinites à La Marsa

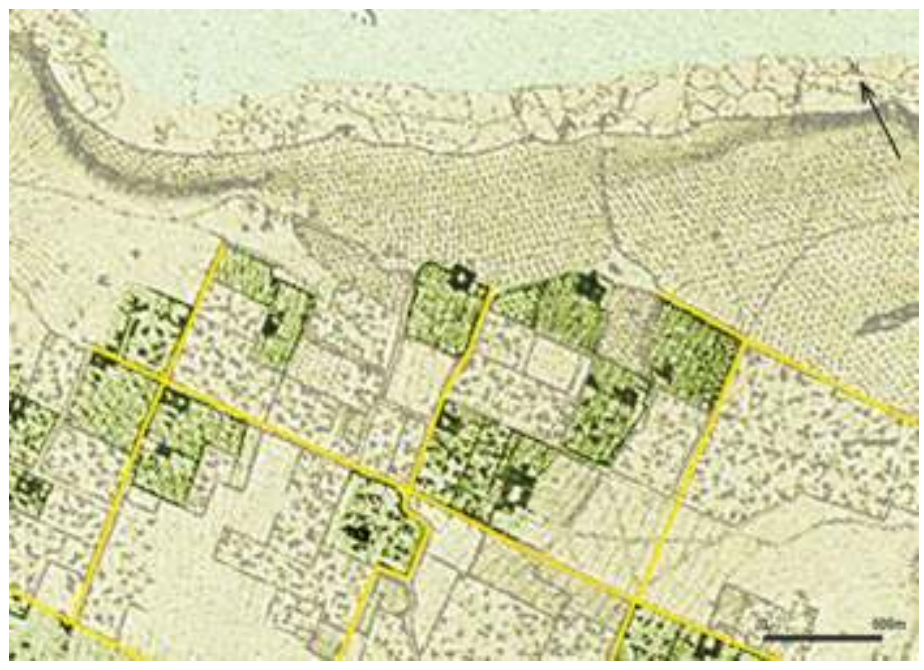

Source : d'après le plan de Carthage dessiné par Falbe en 1831

\section{Le modèle traditionnel}

C'est à Sidi Bou Saïd qu'on rencontre le modèle traditionnel (figure 6). Les résidences secondaires sont très proches les unes des autres et forment un tissu très dense qui rappelle celui de la médina. La hiérarchie spatiale de la médina fait que le repère central est la mosquée. Ainsi, la centralité de la cité est religieuse : tout s'ordonne autour du lieu de culte (Santelli, 1995). De même à Sidi Bou Saïd, nous constatons la même organisation avec un repère central et religieux qui est la zaouïa du saint.

Figure 6. Morphologie urbaine de Sidi Bou Saïd au xxe siècle

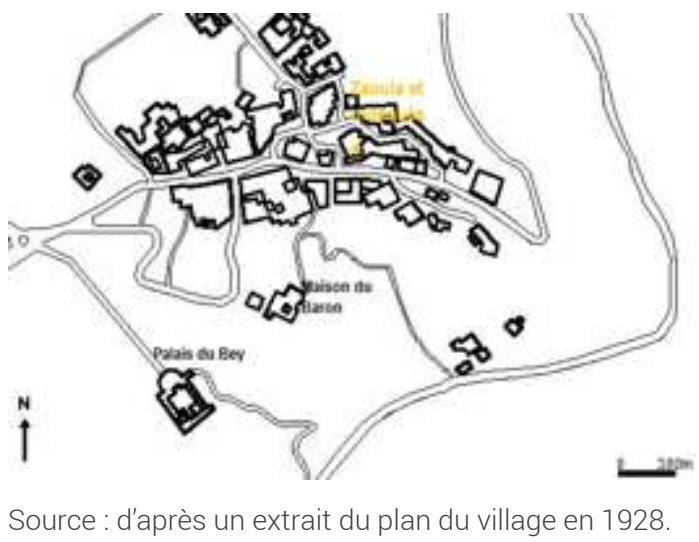

Dans ce modèle, l'organisation de l'espace obéit aux règles de l'organisation de la ville arabo-islamique (médina), où chaque quartier urbain représente à lui seul un espace très complexe régi par la structure familiale ${ }^{7}$. Une autre caractéristique des résidences estivales à Sidi Bou Saïd est d'ouvrir généralement sur des impasses et rarement sur les principaux axes de circulation. Les résidences sont ainsi très proches les unes des autres et forment une masse compacte entourée par des vergers et des parcelles agricoles comme le montre cette vue de Sidi Bou Saïd au début du xxe siècle (figure 7). 
Figure 7. La vue de Sidi Bou Saïd par le voyageur Camille Mauclaire, photo datant de 1910

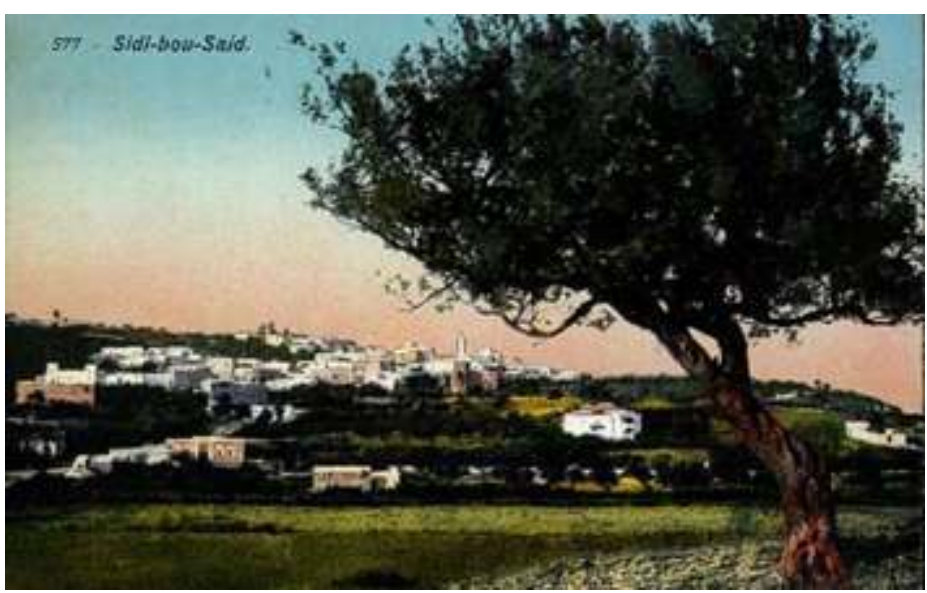

Source : dans Les Douces Beautés de la Tunisie, Paris, Grasset, 1936.

\section{Le modèle en damier}

En s'installant en Tunisie à la fin du XIX $x^{e}$ siècle, les colons français importent leur propre modèle urbain avec la création de villes balnéaires coloniales, comme Hammam-Lif, conçues sur le modèle deauvillois (quadrillage égalitaire destiné à la juxtaposition de pavillons individuels). Dans sa thèse, Roland Vidal (2003) explique que «ce principe du plan orthogonal marquant la domination d'une civilisation conquérante et la négation de la culture antérieure sera appliqué, avec plus ou moins de brutalité, dans la plupart des villes coloniales, entre le $\mathrm{xvI}^{\mathrm{e}}$ et le $\mathrm{xx}^{\mathrm{e}}$ siècle ».

Le nouveau tracé régulier introduit par les Français va concerner des territoires qui étaient, jusqu'à l'occupation française, presque vides de population. Le but de cette organisation était de permettre à toutes les villas d'avoir un accès à la mer le plus proche possible. L'aménagement débuta dans certains cas avec une première rangée de résidences secondaires parallèle à la ligne de côte (exemple d'Ezzahra, autrefois appelé Saint-Germain), ou par le tracé de deux axes principaux perpendiculaires qui définiront plus tard l'organisation de toute la station (c'est le cas de Hammam-Lif). L'ensemble de la station se déploie autour de la gare et du casino sans vraiment favoriser une quelconque centralité (figure 8). 
Figure 8. La morphologie urbaine de la station balnéaire de Hammam-Lif, début du $x x^{e}$ siècle

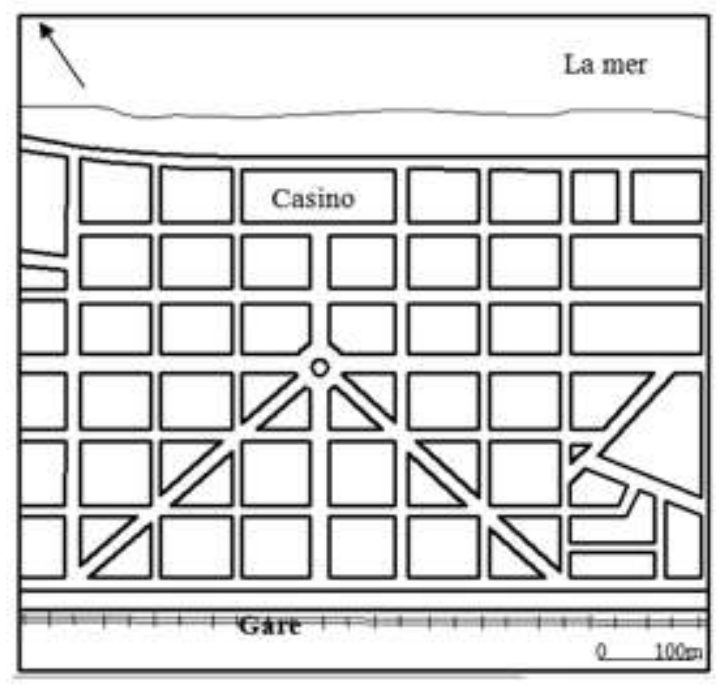

Ainsi, dès l'apparition de la première station balnéaire sur les côtes sud, d'autres lieux subissent les effets de la promotion balnéaire et se développent aussi rapidement et selon un plan similaire.

À partir des années 1920, le lotissement régulier devient le modèle de référence. Il sera parfois très simplifié comme à Ezzahra (figure 9) et peut, dans d'autres cas, donner naissance à un plan en étoile qui permet des variations parcellaires plus fines, comme celui du Kram (figure 10).

Figure 9. Le plan régulier d'Ezzahra (Saint-Germain) et le plan en étoile du Kram
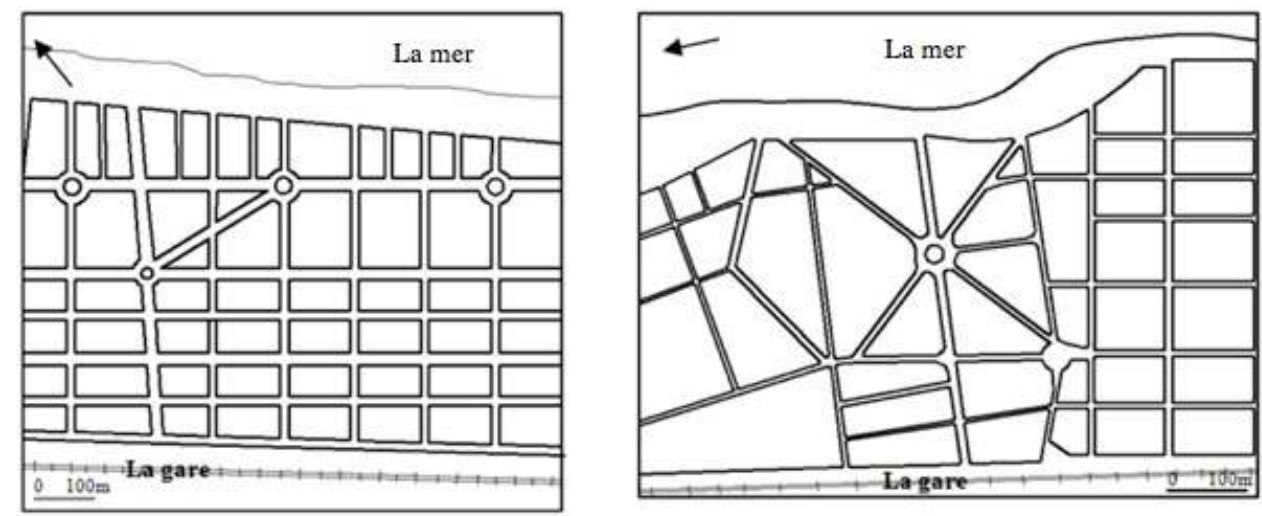

Le point de convergence entre ces différentes stations et les réseaux qui les relient est la création d'un maillage du territoire pour l'occupation d'espaces vierges consacrés à l'activité balnéaire.

\section{Conclusion}

Il ressort ainsi de cette typologie une grande différence entre, d'un côté, les stations de villégiature datant de l'époque coloniale (modèle importé), ayant fait l'objet d'un projet organisé et réfléchi, et d'un autre, les lieux de villégiature littorale datant du XvIII ${ }^{\mathrm{e}}$ et $\mathrm{XIX}^{\mathrm{e}}$ siècle (figure 11). Les lieux de villégiature aménagés par les Tunisiens sous 
l'impulsion des souverains husseinites se caractérisent par une édification progressive, qui suggère l'absence d'une volonté organisatrice. Nous les avons classés en deux types : ceux qui s'inspirent de l'organisation de la médina (modèle traditionnel) et ceux qui se basent sur un regroupement familial des résidences estivales (modèle familial).

Figure 10. Schémas de synthèse des différents modèles urbains des lieux de villégiature balnéaire aux $\mathrm{XIX}^{\mathrm{e}}$ et $\mathrm{XX}^{\mathrm{e}}$ siècles dans la région de Tunis

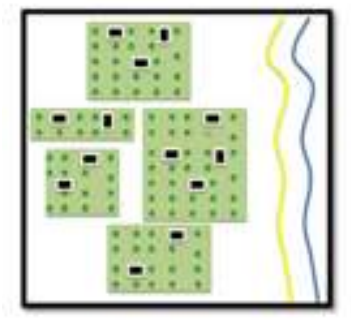

Modele beylical 'familial'

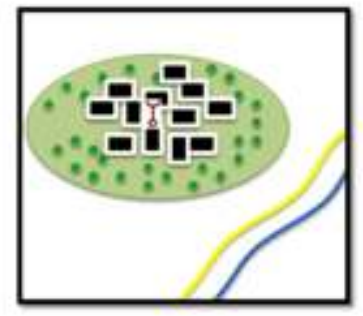

Modèle traditionnel

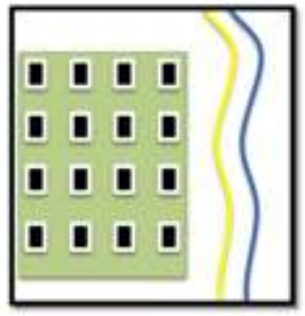

Modele importe

\section{BIBLIOGRAPHIE}

Ben Achour, M., Zaouias et confréries. Aspects de l'islam mystique dans l'histoire tunisienne, Tunis,

Sagittaire Éditions, 2004.

Bergaoui, M., Tourisme et Voyages en Tunisie, les années Régence, 3e édition,Tunis, Simpact, 2005.

Boyer, M., Les Villégiatures du XVI $I^{e}$ au XXI siècle - Panorama du " tourisme sédentaire », Cormelles-leRoyal, EMS Éditions, coll. «Questions de société », 2008.

Brunschvig, R., Deux récits de voyage inédits en Afrique du Nord au Xve siècle: Abdalbasit B. Halil et Adorne, Paris, Maisonneuve \& Larose, 2001.

Corbin, A., Le Territoire du vide. L'Occident et le désir de rivage (1750-1840), Paris, Aubier, coll.

« Historique », 1988.

Devillers, CH., « Typologie de l'habitat et morphologie urbaine », dans Dunant, H., Notice sur la Régence de Tunis, Tunis, Société tunisienne de Diffusion, 1958.

L'Africain, L., Description de l'Afrique (1517), Paris, Maisonneuve, Librairie d'Amérique et d'Orient, 1956.

Largueche, D., « Loisirs, sociabilité et mutations culturelles dans la Régence de Tunis à l'époque ottomane ", Arab Historical Review For Ottoman Studies, n² 29, 2004.

Mahjoub, N., «Sidi Abu Said, le raïs des mers, l'homme, le monument », Tunis cité de la mer, Tunis, édition L'or du temps, 1999, actes du colloque organisé dans le cadre des manifestations relatives au choix de l'Unesco de Tunis, capitale culturelle.

Revault, J., Palais et Demeures de Tunis (XVI et XVII siècles), Paris, Éditions du Centre national de recherches scientifiques, 1980. 
Rey, A. (dir.), Dictionnaire culturel en langue française, Paris, Le Robert, 2005, p. 1914.

Santelli, S., Tunis, le creuset méditerranéen, Paris, édition du Demi-Cercle/CNRS, coll. « La Ville », 1995.

Urbain, J.-D., Sur la plage. Mœurs et coutumes balnéaires (XIXe-XXe siècles), Paris, Payot et Rivages, 2002.

Vidal R., « La construction paysagère d'une identité territoriale. Imaginaire et réalité dans une station balnéaire des Côtes-d'Armor : Sables-d'Or-les-Pins », thèse de doctorat en sciences de l'environnement, Engref Paris mars 2003, p. 51.

Zbiss, M. S., Sidi Bou Saïd, promontoire du soleil éternel, Tunis, Société tunisienne de diffusion, 1971.

\section{NOTES}

1. Bey : titre attribué aux rois de Tunis durant le règne de la dynastie husseinite (1705-1957).

2. Thèse de Zaïer, S., «Évolution de la villégiature sur le littoral nord-est tunisien : modèles nationaux et modèles importés d'urbanisation balnéaire. Pratiques sociales et conséquences paysagères ", ISA de Chott Mariem (Tunisie) et'AgroParisTech/ENSP Versailles (France), mars 2010.

3. La dynastie hafside a régné en Tunisie de 1228 au 1574.

4. Le choix des saints s'était d'abord porté sur la colline de Carthage, avec le saint patron de Tunis, Sidi Mahrez Ibn Khalaf; puis sur la colline de Jbel el Menar, avec Sidi Bou Saïd El Beji.

5. La villégiature en Tunisie trouve ses origines sous les Hafsides dont les pratiques sont adoptées ensuite par la dynastie husseinite puis par les colons français qui y forgent et généralisent les pratiques balnéaires.

6. Sur chaque parcelle occupée par un père de famille apparaitront d'autres maisons de plaisance appartenant à ses proches pour constituer un domaine de la grande famille. C'est le cas du domaine de la couronne à La Marsa.

7. Le modèle urbanistique est la projection de l'organisation sociale codifiée, selon les types complexes des rapports humains, et propre à la civilisation islamique. Voir à ce propos : Daouletli, A., Binous, J. et Lesage, D., «Les opérations intégrées de restructuration urbaine. L'exemple de la médina de Tunis », Monumentum, vol. 25, 1982, p. 3.

\section{RÉSUMÉS}

La transformation des côtes de la région de Tunis en un littoral attractif, émaillé de quelques lieux de villégiature, est le fruit de conditions socioculturelles et économiques particulières. Le changement des perceptions et des mentalités nécessaire à la construction de ces lieux de loisirs s'est opéré progressivement sur quelques sites privilégiés entre la première moitié du XVIII ${ }^{\mathrm{e}}$ siècle et la fin $\mathrm{du} \mathrm{XIX}^{\mathrm{e}}$ siècle. En moins d'un siècle, des stations balnéaires très convoitées se sont développées, accompagnées d'une frénésie pour la plage et les bains de mer. L'expression de ce nouvel engouement s'est manifestée en Tunisie par la construction de palais de plaisance, de résidences estivales, puis de fronts urbains littoraux. Ces nouvelles implantations ont été faites 
par étapes donnant naissance à plusieurs modèles urbains et transformant une région abandonnée en des embryons puis en de véritables lieux d'une villégiature bourgeoise ou populaire estivale.

The transformation of the coasts of the region of Tunis into an attractive shoreline, with a few holiday resorts, is the result of particular socio-cultural and economic conditions and circumstances. Changes in both perceptions and mentalities, needed to build these places of leisure activities, took place gradually in selected and privileged sites during the period between the first half of the eighteenth century and the late nineteenth century. Within less than a century, highly coveted seaside resorts have been developed associated with a new passion for sea bathing and beach activities. This enthusiasm was illustrated in Tunisia by the construction of many recreational palaces and summer residences. These new settlements, built according to a step-by-step process, have generated several urban models transforming an abandoned area to an authentic touristic and resort destination for the upper middle classes as well as a popular place for summer leisure.

\section{INDEX}

Mots-clés : villégiature, urbanisme balnéaire, modèle paysager, littoral tunisois

Keywords : holiday resort, urban resort, landscape model, Tunis coastline

\section{AUTEURS}

\section{SONDÈS ZAIER}

Ingénieur paysagiste diplômé de l'Institut supérieur d'agronomie de Chott Mariem, il est titulaire du master « Paysage, Territoire, Patrimoine » (ISA de Chott Mariem, université de Sousse).

Docteur en sciences agronomiques et en sciences et en architecture de paysage, sa thèse a été codirigée par Pierre Donadieu et Hichem Rejeb, en cotutelle avec l'école doctorale Abies d'AgroParisTech. sondes.zaier[at]laposte[dot]net

\section{HICHEM REJEB}

Maître de conférences, il est directeur de l'unité de recherche « Horticulture, Paysage et Environnement » à l'Institut supérieur d'agronomie de Chott Mariem, université de Sousse. hrejeb62[at]yahoo[dot]fr

\section{PIERRE DONADIEU}

Professeur à l'École nationale supérieure de paysage de Versailles-Marseille. p.donadieu[at]icloud[dot]com 